\title{
1 Pollinators mediate floral microbial diversity and network under agrochemical disturbance
}

3 Na Wei ${ }^{1,2^{*}}$, Avery L. Russell ${ }^{2,3^{*}}$, Abigail R. Jarrett ${ }^{2}$, Tia-Lynn Ashman ${ }^{2}$

$4 \quad{ }^{1}$ The Holden Arboretum, Kirtland, OH 44094, USA.

$5 \quad{ }^{2}$ Department of Biological Sciences, University of Pittsburgh, Pittsburgh, PA 15260, USA.

$6 \quad{ }^{3}$ Department of Biology, Missouri State University, Springfield, MO 65897, USA.

7 *These authors contributed equally: Na Wei, Avery L. Russell

9 Corresponding Author

$10 \quad \mathrm{Na}$ Wei

11 The Holden Arboretum, Kirtland, OH 44094, USA

12 Phone: 440-602-3831; E-mail: nwei@holdenfg.org

13 ORCID: 0000-0002-7345-501X

\section{Keywords}

16 Agrochemical disturbance, bacterial and fungal communities, floral microbiome, microbial

17 network, pollinator visitation, strawberry

\section{Author Contributions}

20 NW, ALR and TLA conceived the study. NW analyzed the data and wrote the manuscript. ALR

21 and TLA contributed to manuscript revision. NW, ALR and ARJ collected the data.

\section{Acknowledgements}

24 This work was funded by the Dr. Frank J. Schwartz Early Career Research Fellowship and G.

25 Murray McKinley Research Fund from the Pymatuning Laboratory of Ecology (NW and ALR)

26 and UPitt Dietrich School of Arts and Sciences (TLA). We thank L. Follweiler for greenhouse

27 and agrochemical application assistance, N. Streher, E. O'Neill, R.A. Hayes, D. Chang, N.

28 Russo, J. Xia, E. James and N. Cullen for assistance in the field and DNA extraction, C.

29 Richards-Zawacki, C.J. Davis and J.A. Barabas for logistic support at the PLE field station. 


\section{Abstract}

33 How pollinators mediate microbiome assembly in the anthosphere is a major unresolved question

34 of theoretical and applied importance in the face of anthropogenic disturbance. We addressed

35 this question by linking visitation of diverse pollinator functional groups (bees, wasps, flies,

36 butterflies, beetles, true bugs and other taxa) to the key properties of floral microbiome

37 (microbial $\alpha$ - and $\beta$-diversity and microbial network) under agrochemical disturbance, using a

38 field experiment of bactericide and fungicide treatments on cultivated strawberries that differ in

39 flower abundance. Structural equation modeling was used to link agrochemical disturbance and

40 flower abundance to pollinator visitation to floral microbiome properties. Our results revealed

41 that (1) pollinator visitation influenced the $\alpha$ - and $\beta$-diversity and network centrality of floral

42 microbiome, with different pollinator functional groups affecting different microbiome

43 properties; (2) flower abundance influenced floral microbiome both directly by governing the

44 source pool of microbes and indirectly by enhancing pollinator visitation; and (3) agrochemical

45 disturbance affected floral microbiome primarily directly by fungicide, and less so indirectly via

46 pollinator visitation. These findings improve the mechanistic understanding of floral microbiome

47 assembly, and may be generalizable to many other plants that are visited by diverse insect

48 pollinators in natural and managed ecosystems.

Introduction

52 Microbiomes can profoundly influence plant fitness in natural and agricultural settings

53 (Berendsen, Pieterse, \& Bakker, 2012; Bulgarelli, Schlaeppi, Spaepen, Ver Loren van Themaat,

54 \& Schulze-Lefert, 2013; Vorholt, 2012). Relative to rhizosphere (root) and phyllosphere (leaf),

55 the anthosphere (floral) microbiome is least well studied but has the most direct impact on plant

56 reproductive success (Aleklett, Hart, \& Shade, 2014; Rebolleda-Gómez et al., 2019; Wei \&

57 Ashman, 2018). Our understanding of what governs microbiome assembly in flowers - a highly

58 dynamic niche - has advanced only recently (Aleklett et al., 2014; Vannette, 2020). The

59 identified drivers include, for instance, microbial dispersal mediated by pollinators (Rebolleda-

60 Gómez \& Ashman, 2019; Vannette \& Fukami, 2017), floral traits that can influence niche

61 availability (e.g. flower size, nectar volume) (Vannette, 2020; Vannette, Hall, \& Munkres, 2020)

62 and microbial source pool (e.g. flower abundance; this study), and disturbance imposed by 
63 bactericides and fungicides especially in crop plants (Bartlewicz, Pozo, Honnay, Lievens, \&

64 Jacquemyn, 2016; Schaeffer, Vannette, Brittain, Williams, \& Fukami, 2017). Yet, as these

65 different drivers have often been studied independently, we lack a clear view as to how they act

66 together and thus their relative importance in shaping floral microbiome.

Pollinator-mediated microbial dispersal is an important determinant of floral microbiome (Rebolleda-Gómez \& Ashman, 2019; Vannette, 2020; Vannette \& Fukami, 2017). Pollinators facilitate microbe colonization and transport but also impact microbial communities by consuming resident microbes (Herrera, Pozo, \& Medrano, 2013; Russell, Rebolleda-Gómez, Shaible, \& Ashman, 2019; Vannette, Gauthier, \& Fukami, 2013). The net outcome on floral microbiome may differ among pollinator taxa (or flower visitors more generally), given their different uses of flowers and preferences for floral resources (Armbruster, 2017; Kantsa et al., 2018; Wei et al., 2020) and microbes (e.g. bacteria vs. yeasts) (Good, Gauthier, Vannette, \& Fukami, 2014; Schaeffer, Rering, Maalouf, Beck, \& Vannette, 2019), as well as different likelihoods of dispersing microbes based on level of interaction with flowers or flower compartments (Russell et al., 2019) or of bringing different microbes to the flowers owing to their remarkably different lifestyles that could affect associated microbes (Engel et al., 2016;

80 Kwong et al., 2017). Research on the effect of pollinators on floral microbiome has been focused on a few pollinator taxa (e.g. honeybees, bumblebees and hummingbirds) (Good et al., 2014; Schaeffer et al., 2019; Vannette et al., 2013), while most plant species are pollinated by diverse groups of pollinator species (Kantsa et al., 2018; Wei et al., 2020). Thus significant knowledge gaps exist as to how different pollinators govern floral microbiome assembly, and further how the effect of pollinators is modulated by other factors that can influence pollinators and the floral

86 microbiome, such as floral traits (e.g. flower abundance) and external disturbance of bactericides 87 and fungicides.

89 Floral traits can influence floral microbiome directly by affecting niche availability and imposing

90 habitat filtering for microbes (Rebolleda-Gómez \& Ashman, 2019; Vannette, 2020; Wei \& 91 Ashman, 2018) and indirectly by affecting pollinator visits (Armbruster, 2017; Kantsa et al.,

92 2018; Wei et al., 2020) that can mediate microbial dispersal. In particular, flower abundance has 93 rarely been studied in floral microbiome but can potentially influence a given flower's microbial 
94 community by affecting the source pool of microbes hosted by neighboring flowers. Such source

95 pool of microbes is dynamic due to rapid habitat extinction (short flower longevity) and

96 temporally shifted habitat availability (changes in flower abundance over time) (Aleklett et al.,

97 2014). Thus flower abundance may directly influence the abundance and richness of microbes

98 that colonize individual flowers independently of pollinators (e.g. by wind or rain splash). In

99 addition, flower abundance can signal resource availability to pollinators (Benadi \& Pauw, 2018;

100 Wei et al., 2020), and thus large displays attract more visits and can potentially affect

101 microbiome indirectly by enhancing microbial dispersal to flowers within the display. Therefore,

102 flower abundance and pollinators act together in shaping floral microbiome, and their

103 independent and interactive effects on floral microbiome remain to be quantified.

105 Theory predicts that disturbance alters niche availability, the nature of species interactions

106 (positive, facilitation or negative, competition) and species composition of communities

107 (Mouillot, Graham, Villeger, Mason, \& Bellwood, 2013). For microbiomes in an agricultural

108 setting, an apparent disturbance can come from bactericides and fungicides that broadly or

109 selectively target microbial taxa (Matson, Parton, Power, \& Swift, 1997; McManus, Stockwell,

110 Sundin, \& Jones, 2002). Chemically-mediated disturbances are becoming more common in the

111 Anthropocene as agrochemical use is increasing, and can reduce the abundance and richness of

112 plant-associated microbes, shift microbial composition (Bartlewicz et al., 2016; Schaeffer et al.,

113 2017) and potentially rewire microbe-microbe interactions via physical and metabolic

114 mechanisms (e.g. habitat sharing via fungal hyphae and bacterial biofilm and by-product cross-

115 feeding) (Deveau et al., 2018; Frey-Klett et al., 2011; Goldford et al., 2018; Smith, Shorten,

116 Altermann, Roy, \& McNabb, 2019). Agrochemical disturbance has also shown off-target effects

117 on pollinators including altering their visitation patterns (Fisher, Coleman, Hoffmann, Fritz, \&

118 Rangel, 2017; Park, Blitzer, Gibbs, Losey, \& Danforth, 2015; Stejskalová, Konradyová,

119 Suchanová, \& Kazda, 2018). Thus, agrochemical disturbance of bactericides and fungicides

120 likely affects flower microbiome both directly (by reducing targeted microbial taxa) and

121 indirectly (by influencing pollinator visitation). It remains, nevertheless, a major unresolved

122 question whether the effect of agrochemical disturbance is generalizable across taxonomically

123 diverse pollinators and between bacterial and fungal constituents of floral microbiome. 
125 Here we used a field experiment to quantify how insect pollinators govern the key properties of

126 floral microbiome (i.e. microbial $\alpha$ - and $\beta$-diversity and microbial network) under agrochemical

127 disturbance (bactericide and fungicide; Figure 1). We focused on the epiphytic floral microbiome

128 of cultivated strawberry (Fragaria $\times$ ananassa). Strawberry crops are commercially important

129 worldwide (FAOSTAT, 2020) and rely heavily on pesticide use for productivity (Environmental

130 Working Group, 2020). While the plant is capable of autonomous self-pollination, visitation by a

131 wide array of insects is essential for pollination success and marketable fruit production (Klatt et

132 al., 2014). Thus strawberry represents a model system to investigate how agrochemicals and

133 pollinators affect microbiome in flowers. Rather than manipulating pollinator access (e.g.

134 Rebolleda-Gómez \& Ashman, 2019; Vannette \& Fukami, 2017), we linked the natural variation

135 in visitation of different functional groups of pollinators (bees, wasps, flies, butterflies, beetles,

136 true bugs and other taxa) to bacterial and fungal $\alpha$ - and $\beta$-diversity and microbial network in

137 flowers. To minimize habitat filtering due to host variation, our experiment used clonal replicates

138 of four genotypes of strawberry. The four genotypes differ dramatically in flower abundance but

139 are similar in other floral traits. To test for the effect of agrochemical disturbance, we performed

140 a replicated factorial design using broad-spectrum bactericide and fungicide at the agricultural

141 application rate (Figure 1a). By doing so, we first characterized the communities of visiting

142 pollinators and floral microbiota (bacteria and fungi), and examined separately how they

143 responded to agrochemical disturbance and plant genotype. We further constructed microbial

144 correlation networks to infer intra-kingdom (bacteria-bacteria and fungi-fungi) and inter-

145 kingdom (fungi-bacteria) interactions among microbes and the network centrality of floral

146 microbiome. Lastly, to link pollinator communities to microbial communities, we used structural

147 equation modeling to quantify how agrochemical disturbance and genotypic variation in flower

148 abundance affected the visitation of pollinator functional groups and thereby together affected

149 microbiome properties ( $\alpha$ - and $\beta$-diversity and network centrality; Figure $1 \mathrm{~b}$ ). These analyses

150 revealed that (1) pollinator visitation influenced floral microbiome with different pollinator

151 functional groups affecting different properties of floral microbiome; (2) flower abundance

152 strongly affected floral microbiome both directly and indirectly via enhancing pollinator

153 visitation; (3) agrochemical disturbance affected floral microbiome primarily directly, and the

154 effect was caused by fungicide rather than bactericide. 
Materials and Methods

157 Experimental design

Strawberry plant system and factorial experimental design

159 We used four day-neutral strawberry cultivars (Mara Des Bois, Albion, Portola, San Andreas;

160 Nourse Farms, Whately, MA) that show pronounced differences in flower production (Figure

161 2a). For each genotype (cultivar), we planted 80 clonal 'bare root' plants in 2.4 L pots filled with

162 Fafard 4 mix (Sun Gro Horticulture, Agawam, MA) on May 3, 2018. These plants were grown at

$16324^{\circ} \mathrm{C}$ day $/ 18^{\circ} \mathrm{C}$ night temperatures 12 -h days in a greenhouse at the University of Pittsburgh for

1642 wk until they were transported to a field at the Pymatuning Laboratory of Ecology, Linesville

165 PA $\left(41.57011^{\circ} \mathrm{N}, 80.45619^{\circ} \mathrm{W}\right)$. Potted plants grew and accumulated natural microbiota for $5 \mathrm{wk}$

166 prior to the start of a 4-wk experiment of agrochemical treatment, pollinator observation, flower

167 abundance survey and floral microbiome collection from June 24 to July 21, 2018. The factorial

168 design of agrochemical treatments included water control (' $\mathrm{C}$ '), bactericide (' $\mathrm{B}$ ', oxytetracycline,

169150 ppm; Sigma-Aldrich, St. Louis, MO), fungicide ('F', azoxystrobin, Heritage, 0.2 lb per acre;

170 Syngenta, Greensboro, NC), and bactericide and fungicide ('BF'). The four treatments were

171 applied at the 'block' level ( $N=40$ with 10 blocks per treatment, Figure 1a). Each experimental

172 block consisted of four pairs of clones (one pair of each genotype) and the location of pairs

173 within block was randomized. Pairs were used to ensure floral display for pollinators and were

174 referred to as one 'plant sample' hereafter (Figure 1a). Treatments were applied weekly using a

175 backpack sprayer, with plant samples receiving an equal volume across treatments each week

176 ('C': $25 \mathrm{~mL}$ water per plant sample; 'B': $12.5 \mathrm{~mL}$ water and $12.5 \mathrm{~mL}$ bactericide; ' $\mathrm{F}$ ': $12.5 \mathrm{~mL}$

177 water and $12.5 \mathrm{~mL}$ fungicide; 'BF': $12.5 \mathrm{~mL}$ bactericide and $12.5 \mathrm{~mL}$ fungicide). Plants were

178 protected from large herbivores using fencing with buffer distance $(10 \mathrm{~m})$ to the site.

180 During the 4-wk experiment, due to temporal dynamics in flower production and pollinator

181 visitation between the first $2 \mathrm{wk}$ and last $2 \mathrm{wk}$ (Figures S1 and S2), we divided the experiment

182 into the corresponding two time periods (Time 1 and Time 2).

\section{Pollinator observation}

185 Pollinator observations were conducted by the same observer four days each week between 186 0900-1700 h. The observer spent 2 min per block for all 40 blocks in a single observation round. 
187 At each block, all flowers were observed simultaneously and the number of pollinators (bees,

188 wasps, flies, butterflies, beetles, true bugs and other taxa) were recorded for each plant sample.

189 Each day 1-3 rounds of observations were completed with the order of block visits

190 systematically alternated each round. As a result, we spent 2874 min total for pollinator

191 observations in this experiment, and reported the visitation data as the number of visiting

192 pollinators per plant sample per $\sim 36$ min during each 2 -wk time period.

\section{Flower abundance survey}

195 We scored the number of flowers including both open flowers and flower buds of each plant

196 sample once per week, and reported flower abundance data as the sum of flowers per plant

197 sample per 2-wk time period. We also confirmed that the four genotypes differed significantly in

198 flower abundance (Figure 2a; SI Methods) and not other traits (e.g. flower size and pollen

199 production, Figure S1; SI Methods) that reflect attraction and resources for pollinators (Ashman, 200 2000).

Floral microbiome collection and sequencing

203 Two flowers were collected from each plant sample each week two days post agrochemical

204 application. Following Wei and Ashman (2018), flowers (without pedicel) were collected into a

205 sterile $15 \mathrm{~mL}$ centrifuge tube using ethanol-rinsed forceps and were transferred to a $-20{ }^{\circ} \mathrm{C}$

206 freezer within two hours. For microbial DNA extraction, we pooled flowers standardized by size

$207(N=1-4)$ per plant sample per 2-wk time period and obtained 246 total samples. Epiphytic

208 microbes were collected by sonicating flowers in $3 \mathrm{~mL}$ phosphate-buffered saline at $40 \mathrm{kHz}$ for

$20910 \mathrm{~min}$ and vortexing for $5 \mathrm{~min}$, and were pelleted by centrifuging at 13,300 rpm for $5 \mathrm{~min}$.

210 Microbial DNA was extracted using Quick-DNA Fecal/Soil Microbe Kits (Zymo Research,

211 Irvine, CA). Two negative controls without flower samples were included in the process of

212 microbe isolation and DNA extraction. Samples and negative controls were sent to Argonne

213 National Laboratory for bacterial (16S rRNA V5-V6 region, 799f-1115r primer pair) (Redford,

214 Bowers, Knight, Linhart, \& Fierer, 2010) and fungal (ITS1f-ITS2) (Smith \& Peay, 2014) library

215 preparation. Because the negative controls failed in PCRs in library preparation, the 246 samples

216 were sequenced on two lanes of Illumina MiSeq paired-end $250 \mathrm{bp}$. 


\section{Bioinformatic and statistical analyses}

219 Microbial sequence processing

220 Demultiplexed paired-end (PE) reads were used for detecting bacterial and fungal amplicon

221 sequence variants (ASVs) using package DADA2 v1.12.1 (Callahan et al., 2016) in R v3.6.0 (R

222 Core Team, 2019) and QIIME 2 v2019.4 (Bolyen et al., 2019). For bacterial ASV analysis in

223 DADA2, PE reads were trimmed and filtered [truncLen $=c(245,245)$, trimLeft $=c(10,0)$, $\operatorname{maxN}$

$224=0$, truncQ $=2$ ] after initial quality inspection. Then end-specific variants were identified after

225 taking into account sequence errors, prior to joining the PE reads (minOverlap $=20$,

226 maxMismatch =4) for ASV detection. Bacterial ASVs were further filtered against chimeras and

227 assigned with taxonomic identification based on the SILVA reference database (132 release)

228 implemented in DADA2. For fungal ASV analysis in DADA2, the PE reads were first screened

229 to remove potential primer contaminations, and then subject to the ASV detection processes from

230 trimming and quality filtering [truncLen $=c(200,200), \operatorname{maxN}=0$, truncQ $=2$ ] to chimera

231 removal, as described for bacteria. Fungal taxonomic assignment was conducted based on the

232 UNITE reference database (v8.0 dynamic release) using QIIME 2.

234 Bacterial and fungal ASV tables were further filtered separately before conversion into microbial 235 community matrices using package phyloseq (McMurdie \& Holmes, 2013). First, we removed 236 non-focal ASVs (Archaea, chloroplasts and mitochondria). Second, we filtered out low-depth 237 samples ( $<100$ reads; $N=13$ and 8 samples for bacterial and fungal data set, respectively). Third, 238 we normalized per-sample reads to the same number (i.e. the median reads, 18788 and 37516, 239 bacterial and fungal data set, respectively) following Wei and Ashman (2018). Lastly, we 240 removed low-frequency ASVs $(<0.001 \%$ of total observations $)$. The final community matrices 241 consisted of 1237 and $1165 \mathrm{ASVs}$ for bacteria $(N=223)$ and fungi $(N=240)$, respectively.

\section{Pollinator visitation and community composition}

244 To evaluate how pollinator visitation responded to agrochemical treatment, genotype and time 245 period, we examined pollinator fauna as a whole and individual functional groups using zero246 inflated generalized linear mixed models (zGLMMs). In each zGLMM, the predictors included

247 treatment (levels: C, B, F and BF), genotype (1: Mara Des Bois; 2: Albion; 3: Portola; 4: San 248 Andreas) and time period (Time 1 vs. Time 2), with random effects being block and plant sample 
249 (due to repeated measurements over time) using package glmmTMB (Brooks et al., 2017).

250 Negative binomial errors were used in zGLMMs and model fits were confirmed using package

251 DHARMa (Hartig, 2019). Statistical significance (type III sums of squares) and least-squares

252 means (LS-means) of predictors were assessed using packages car (Fox \& Weisberg, 2011) and

253 emmeans (Lenth, 2019).

To evaluate how the composition of the pollinator communities responded to agrochemical

256 treatment, genotype and time period, we converted the pollinator functional group matrix into

257 proportional data, and conducted permutational multivariate analysis of variance

258 (PERMANOVA; predictors: treatment, genotype and time period; random effect: block) using

259 package vegan (Oksanen et al., 2019). Once significant predictors were detected, we further

260 identified the specific functional groups underlying pollinator community variation caused by the

261 predictors using random forest (RF) classification in packages caret (Kuhn, 2019) and

262 randomForest (Liaw \& Wiener, 2002). The RF classification models were run for the full data

263 with 1000 trees, and the number of randomly selected variables (i.e. functional groups) at each

264 split of a decision tree was optimized using 10-fold cross validation in caret. RF model

265 performance was assessed using out-of-bag (OOB) error. The set of important variables

266 (functional groups) were selected using backwards variable elimination with package varSelRF

267 (Diaz-Uriarte, 2007), and their relative importance was evaluated using mean decrease in

268 classification accuracy.

Floral microbial $\alpha$ - and $\beta$-diversity

271 To evaluate how microbial $\alpha$-diversity responded to agrochemical treatment, genotype and time

272 period, we calculated Shannon diversity for bacterial and fungal communities separately using

273 vegan, and performed general linear mixed models (LMMs) using package lme4 (Bates,

274 Machler, Bolker, \& Walker, 2015). The predictors included treatment, genotype and time period

275 as well as their two-way interactions, and the random effects included block and plant sample.

276 The response variable was power transformed to improve normality, with the optimal power

277 parameter determined using the Box-Cox method in package car. Statistical significance of

278 predictors in LMMs was evaluated using package lmerTest (Kuznetsova, Brockhoff, \&

279 Christensen, 2017). 
Microbial $\beta$-diversity (Bray-Curtis dissimilarity) was evaluated using PERMANOVA and constrained principal coordinates analysis ( $\mathrm{CPCoA})$ in vegan for bacterial and fungal communities separately. To assess the significance of the main effects, PERMANOVA and

284 cPCoA included treatment, genotype and time period with block random effect. To assess the significance of two-way interactions, PERMANOVA and cPCoA included both the main effects and their interaction terms. Once significant predictors were identified, random forest classification was used to detect the set of important ASVs underlying bacterial or fungal community variation caused by the predictors, as described for pollinator community composition.

Structural equation models (SEMs) linking pollinators to microbial diversity

292 To evaluate how pollinators influenced microbial diversity under agrochemical disturbance, we conducted SEMs to link flower abundance and agrochemical treatment to visitation by pollinators of specific functional groups to $\alpha$ - and $\beta$-diversity of bacteria or fungi in the floral microbiome (Figure 1b). The SEMs included flower abundance as genotypic variation was only significant in this trait (Figure 2a) and not the others (Figure S1). The SEMs were conducted on data collected across both time periods but did not include time period as an exogenous

298 (independent) variable, because time-period variation was defined and reflected by variation in flower abundance and pollinator visitation (Tables S1 and S2). For exogenous categorical

300 variables of treatments, we coded the control treatment as the reference level so that the effects 301 of all other treatments were relative to the control. The endogenous (dependent) variable of the $302 \alpha$-diversity metric (Shannon diversity) was power transformed as in section Floral microbial $\alpha$ 303 and $\beta$-diversity (power parameter $=1$ and 2 for bacteria and fungi, respectively). The endogenous 304 variable of $\beta$-diversity used the first axis of the cPCoA (see section Floral microbial $\alpha$ - and $\beta$ 305 diversity) that accounted for $25 \%$ and $60 \%$ of total variation in bacterial and fungal communities, 306 respectively. Model estimation used robust maximum likelihood with Satorra-Bentler scaled $\chi^{2}$ 307 that can accommodate nonnormality using package lavaan (Rosseel, 2012). Model fits were 308 confirmed (comparative fit index, CFI > 0.9; root mean squared error of approximation, 309 RMSEA, the lower bound of $90 \%$ confidence interval $<0.05$; standardized root mean squared 310 residual, SRMR < 0.1) (Kline, 2015). We conducted the SEMs using the original and square-root 
311 transformed pollinator visitation data separately, which yielded qualitatively similar results but

312 the former exhibited better model fits and thus were reported here. Standardized coefficients $(r)$

313 were indicated for paths with notable effects $(P<0.1)$.

315 Microbial networks and SEMs linking pollinators to network centrality

316 Microbial correlation networks were constructed to infer intra- and inter-kingdom interactions

317 among microbes in flowers, and how pollinators influenced microbial network centrality under

318 agrochemical disturbance. We focused on two centrality metrics (degree and eigenvector). In a

319 network, degree centrality describes a microbe's importance based on the total number of direct

320 connections (or links) with other microbes. Eigenvector centrality weighs each connection by

321 giving higher weights to connections to well-connected (i.e. high degree) microbes and considers

322 both direct and indirect connections, and thus is a weighted sum of all connections of a microbe

323 to other microbes (Bonacich, 2007). Therefore, degree and eigenvector centrality measure the

324 network importance of microbes based on unweighted direct interactions and weighted network-

325 wide interactions, respectively.

327 To build microbial networks, we merged the bacterial and fungal community matrices and 328 normalized per-sample read numbers (to the median of the bacterial data set). This resulted in 3292346 ASVs (1232 bacteria and 1114 fungi) across 218 samples that had data for both bacteria 330 and fungi. To infer positive and negative microbe-microbe interactions, we used SparCC

331 (Friedman \& Alm, 2012) that is robust to spurious correlations among microbes due to

332 microbiome compositionality (i.e. non-independence among ASVs due to relative abundances)

333 using package SpiecEasi (Kurtz et al., 2015). To do this, we extracted the raw counts of these

334 ASVs, and focused on the ASVs that were present in $\geq 10$ samples. Networks were constructed

335 and visualized for each treatment (based on correlation estimates $\geq 0.4$ and $\leq-0.4$ ) using SparCC

336 and package igraph (Csardi \& Nepusz, 2006).

338 To infer how pollinators influenced microbial network (degree and eigenvector centrality) under 339 agrochemical disturbance using SEMs (Figure 1b), we targeted ASVs that were responsive to (1) 340 visitation of pollinator functional groups and (2) flower abundance based on maximal

341 information coefficient (MIC $\geq 0.2$ ) (Reshef et al., 2011) and (3) agrochemical treatments using 
random forest (RF) classification. MICs that can detect nonlinear associations were calculated using package minerva (Albanese et al., 2013). RF classification was performed to detect the ASVs that differed between pairwise comparisons of treatments for bacteria and fungi separately. This resulted in 238 ASVs (146 bacteria and 92 fungi) in the 218 samples for SEMs. To do this, we performed leave-one-out approach to measure network change caused by the removal of a sample. Specifically, we constructed the microbial network with and without a sample based on MIC $(\geq 0.2)$ due to its nonlinear detectability. We then calculated node-level degree and eigenvector centrality for individual nodes (ASVs) using igraph. Network change in node-level centralities was calculated as the distance between two vectors of ASV centralities (i.e. with and without a sample) using package pdist (Wong, 2013) for degree and eigenvector centrality separately. Such network change reflected individual sample's importance to microbial network centrality and was used in the SEMs.

\section{Results}

\section{Pollinator visitation and community composition}

357 The visitation of all pollinator fauna $\left(\chi^{2}=4.7, \mathrm{df}=3, P=0.20, \mathrm{zGLMM}\right)$ and that of individual

358 functional groups (Table S2) were similar among different agrochemical treatments, except

359 butterflies and the 'other' category (Figure S2). Butterfly visitation tended to increase when both

360 bactericide and fungicide were applied (BF vs. C; zGLMM, $t=2.15, P=0.030$, Figure S2b).

361 The 'other' group of pollinators decreased under fungicide treatment relative to the control (F vs.

$362 \mathrm{C} ; t=-2.01, P=0.045$, Figure S2c). Different from agrochemical treatment, plant genotype

363 strongly influenced visitation of all pollinators combined $\left(\chi^{2}=178, \mathrm{df}=3, P<0.001\right.$, Figure $\left.2 \mathrm{~b}\right)$

364 and that of individual functional groups (all $P<0.05$, Table S2). In addition, pollinator visitation

365 declined over time period $\left(\chi^{2}=41.6, \mathrm{df}=1, P<0.001\right.$, Figure $\left.\mathrm{S} 2 \mathrm{a}\right)$, especially for true bugs

366 (Table S2). Similar to visitation, pollinator community composition also varied significantly over 367 time period (PERMANOVA, $F=34.5, \mathrm{df}=1, P=0.001,12 \%$ of variation, Figure $2 \mathrm{c}$ ) and

368 among plant genotypes $(F=2.2, \mathrm{df}=3, P=0.025,2 \%$, Figure $2 \mathrm{c}$ and Figure $\mathrm{S} 2 \mathrm{~d})$, which were

369 driven by changes in the relative abundance of true bugs and flies (random forest, OOB $=21.4 \%$,

370 Table S3; Figure 2c) and that of true bugs and beetles $(\mathrm{OOB}=63.3 \%)$, respectively. 
Agrochemical treatment significantly affected the $\alpha$-diversity of fungal communities (LMM, $F=$ 3.59 , df $=3, P=0.022$, Table S4), after accounting for the effects of genotype $(F=4.09$, $\mathrm{df}=3$, $P=0.009$, Figure S3) and time period $(F=2.04, \mathrm{df}=1, P=0.16)$. As expected, fungal Shannon diversity was reduced when fungicide was applied (F vs. C, $\chi^{2}=7.16$, $\mathrm{df}=1, P=0.007$; BF vs. $\mathrm{C}, \chi^{2}=7.74, \mathrm{df}=1, P=0.005$, Figure $3 \mathrm{c}$ ). This reduction was especially strong at Time 1 (treatment $\times$ time period, $F=4.20, \mathrm{df}=3, P=0.007$ ), whereas at Time 2 all treatments remained low in fungal Shannon diversity including the control (Figure S3b), likely due to the combined effect of agrochemical use and reduced flower abundance (over time period) that had a strong effect on fungal diversity (described below in SEMs).

Unexpectedly, bacterial Shannon diversity was significantly reduced by fungicide (LMM, F vs. $\mathrm{C}, \chi^{2}=5.71, \mathrm{df}=1, P=0.017$; $\mathrm{BF}$ vs. $\left.\mathrm{C}, \chi^{2}=5.34, \mathrm{df}=1, P=0.021\right)$ rather than bactericide (B vs. C, $\chi^{2}=1.97, \mathrm{df}=1, P=0.16$, Figure $3 \mathrm{a}$ and Figure $\mathrm{S} 3 \mathrm{a}$ ). The weak effect of bactericide on bacterial diversity was consistent with the observations of microbial networks (Figure 3e). Bactericide increased positive bacteria-bacteria correlations (B, 522 out of 796 total intra- and inter-kingdom links) relative to the control (399 of 795), whereas fungicide reduced positive bacteria-bacteria (232 and 146 in F and BF vs. 399 in C) and positive fungi-bacteria correlations (12 and 14 in F and BF vs. 43 in C), as well as the total links (432 and 254 in F and BF vs. 795 in C).

Similar to $\alpha$-diversity, fungal community composition was affected by agrochemical treatment, which explained the largest source of variation in fungal communities (PERMANOVA, 15\%, $F$ $=15.2, \mathrm{df}=3, P=0.001$, Table S4) relative to time period $(5 \%, F=15.9, \mathrm{df}=1, P=0.001)$ and plant genotype $(3 \%, F=2.7, \mathrm{df}=3, P=0.001)$. The cPCoA further revealed that fungicide caused the divergence of fungal communities of $\mathrm{F}$ and $\mathrm{BF}$ treatments from the control (along the first axis, cPCoA1, Figure 3d). This divergence was driven by a small set of ASVs (F vs. C, $N=$ $40, \mathrm{OOB}=11.5 \%$; BF vs. $\mathrm{C}, N=27, \mathrm{OOB}=9.2 \%$ ), among which Basidiomycota and Ascomycota fungi exhibited the largest importance (Figure S4 and Table S5). In particular, Rhodotorula graminis (yeast) was significantly enriched, and Sporobolomyces phaffii (yeast) and

402 Cladosporium delicatulum (mold) were significantly depleted in F and BF treatments relative to 403 the control (adjusted $P$ for multiple comparisons $<0.001$, Table S5). 
Different from fungi, bacterial communities were primarily segregated over time period along $\operatorname{cPCoA} 1(F=6.96, \mathrm{df}=1, P=0.001$, Figure 3b; PERMANOVA, $F=7.62, P=0.001$, Table S4). Albeit subtler than fungi (Figure 3b,d), bacterial communities varied among agrochemical treatments (cPCoA, $F=1.38, \mathrm{df}=3, P=0.017$; PERMANOVA, $F=1.44, P=0.015$, Table S4), with all other treatments (B, F and BF) significantly deviated from the control (PERMANOVA, pairwise comparisons, all $P<0.05$; data not shown). Such deviation was driven by a small set of $\operatorname{ASVs}(N=8,14$ and 3 for B, F and BF, respectively, Table S5), among which two Hymenobacter ASVs were most influential (Figure S5) and significantly depleted in other treatments relative to the control (adjusted $P<0.001$, Table S5).

\section{Pollinators influenced floral microbiome}

416 SEMs revealed that pollinator functional groups varied in their influence on different properties 417 of floral microbiome ( $\alpha$ - and $\beta$-diversity and network centrality), independent of the significant 418 direct effects of flower abundance and agrochemical treatments (Figure 4). Bee visitation had a 419 notable positive effect on bacterial Shannon diversity $(r=0.12, P=0.070$, Figure $4 \mathrm{a})$ but a 420 negative effect on fungal Shannon diversity $(r=-0.13, P=0.068$, Figure $4 \mathrm{~b})$, whereas butterfly 421 visitation tended to reduce bacterial Shannon diversity $(r=-0.08, P=0.065)$. In contrast to $\alpha-$ 422 diversity, the $\beta$-diversity of microbial communities (cPCoA1) was influenced by a different set

423 of pollinators (Figure 4c,d). Visitation of flies and butterflies showed contrasting effects relative 424 to true bugs on bacterial ( $r=0.15$ and 0.11 vs. $-0.17, P=0.015,0.046$ and 0.036 , respectively, 425 Table S6) and fungal community composition $(r=0.19$ and 0.12 vs. $-0.20, P<0.001, P=0.011$ 426 and $P<0.001$, respectively). Different from $\alpha$ - and $\beta$-diversity, pollinator visitation did not affect 427 microbial network degree centrality (Figure 4e). But visitation of bees and flies influenced 428 eigenvector centrality ( $r=-0.09$ and $-0.17, P=0.083$ and 0.014 , respectively, Figure $4 \mathrm{f}$ ). Flower abundance affected floral microbiome directly and indirectly

431 SEMs revealed that flower abundance affected floral microbiome both directly and indirectly via 432 influencing pollinator visitation (Figure 4). Flower abundance had a direct positive effect on 433 fungal Shannon diversity $(r=0.23, P=0.024$, Figure $4 \mathrm{~b})$, and also directly affected microbial 434 community composition (bacteria, $r=-0.28, P=0.011$, Figure $4 \mathrm{c}$; fungi, $r=-0.13, P=0.088$, 
435 Figure 4d) and network centrality (degree, $r=0.33, P=0.006$, Figure $4 \mathrm{e}$; eigenvector, $r=0.44$,

$436 P<0.001$, Figure 4f). In addition, flower abundance affected microbiome indirectly by

437 increasing pollinator visitation consistently across all functional groups (all $P<0.05$, Figure 4).

438 In particular, bacterial Shannon diversity was affected by flower abundance only indirectly via

439 pollinators [bees, $r=0.06(0.52 \times 0.12)$; butterflies, $r=-0.02(0.19 \times-0.08)$, Figure 4a], whereas

440 fungal Shannon diversity was affected by flower abundance both directly and indirectly [bees, $r$

$441=-0.06(0.49 \times-0.13)$, Figure $4 b]$, similar to other properties of microbiome (community

442 composition and eigenvector centrality). The indirect effect of flower abundance was similar

443 between bacterial community composition [flies, $r=0.08(0.56 \times 0.15)$; butterflies, $r=0.02$

$444(0.19 \times 0.11)$; beetles, $r=-0.02(0.26 \times-0.09)$; true bugs, $r=-0.10(0.60 \times-0.17)$, Figure $4 \mathrm{c}]$ and

445 fungal community composition [flies, $r=0.10(0.53 \times 0.19)$; butterflies, $r=0.02(0.18 \times 0.12)$;

446 true bugs, $r=-0.12(0.60 \times-0.20)$, Figure $4 d]$. For network eigenvector centrality (Figure $4 \mathrm{f})$, the

447 indirect effect of flower abundance was via bees $[r=-0.05(0.52 \times-0.09)]$ and flies $[r=-0.09$

$448(0.55 \times-0.17)]$.

\section{Agrochemical disturbance affected floral microbiome primarily directly by fungicide}

451 While the significant effect of agrochemical treatment on microbiome described above (LMMs, 452 PERMANOVA/cPCoA and network links, Figure 3) reflected the overall effect of agrochemical 453 treatment, SEMs dissected the overall effect into direct vs. indirect effect (Figure 4). The indirect 454 effect of agrochemical treatment (B, F and BF) via pollinators was weak, because pollinator 455 visitation was rarely affected by agrochemical treatment except for butterflies $(r=0.25, P<$ 4560.05 , Figure 4$)$ and the 'other' group $(r=-0.14, P<0.10)$ consistent with the zGLMMs results 457 above, as well as true bugs (SEMs, $r=0.10, P<0.10 ; \mathrm{zGLMM}, t=1.67, P=0.097$ ). The 458 indirect effect of agrochemical treatment was only present via butterflies on bacterial Shannon 459 diversity $[\mathrm{B}, r=-0.01(0.09 \times-0.08)$; BF, $r=-0.02(0.25 \times-0.08)$, Figure $4 \mathrm{a}]$ and community 460 composition $[\mathrm{B}, r=0.01(0.09 \times 0.11)$; BF, $r=0.03(0.25 \times 0.11)$, Figure $4 \mathrm{c}]$, and on fungal 461 community composition via butterflies [B, $r=0.01(0.08 \times 0.12)$; BF, $r=0.03(0.25 \times 0.12)$, 462 Figure $4 \mathrm{~d}]$ and true bugs $[\mathrm{F}, r=-0.02(0.10 \times-0.20)]$. In contrast to the weak indirect effect, the 463 direct effect of agrochemical treatment was strong for all measured microbiome properties and 464 for both bacteria and fungi (all $P<0.05$, Figure 4). Similar to the overall effect revealed by other 
methods (LMMs, PERMANOVA/cPCoA and network links, Figure 3), the direct effect of agrochemical treatment on microbiome was driven by fungicide (F and BF, Figure 4).

\section{Discussion}

469 Disentangling potentially interacting mechanisms in shaping microbiomes is critical for theoretical and applied advances in the principles of microbiome assembly (Hawkes \& Connor, 2017). Our work demonstrated the role of taxonomically diverse insect pollinators, and their interactions with other drivers in shaping the properties of floral microbiome. Pollinator functional groups showed distinct effect on microbial $\alpha$ - and $\beta$-diversity and network centrality.

474 The effect of pollinator visitation was strongly influenced by flower abundance but less so by

475 agrochemical disturbance. Instead, agrochemical disturbance primarily from fungicide directly

476 affected both bacterial and fungal communities. By linking these mechanisms previously studied

477 in isolation (see refs in Introduction), our results provide an integrated understanding of the

478 drivers and their respective importance in governing microbiome assembly in the anthosphere in

479 the face of agrochemical disturbance.

Our results revealed that pollinator functional groups influenced bacterial and fungal communities, even after accounting for the strong direct effects of flower abundance and agrochemical disturbance. In particular, the most abundant functional groups that visited

484 strawberry flowers (bees, flies and true bugs, Figure 2c) played the most important roles in 485 governing microbial diversity relative to other pollinators. These abundant pollinators differed in respective effects on microbial $\alpha$ - vs. $\beta$-diversity (Figure 4), with bee visitation influencing $\alpha$ -

487 diversity and true bugs and flies influencing $\beta$-diversity. These distinct effects may reflect 488 different rates of microbial dispersal mediated by different functional groups. Microbial dispersal 489 via bees, the most important insect pollinators of cultivated strawberry (Klatt et al., 2014), can be 490 potentially extensive and thus increase $\alpha$-diversity (e.g. in bacterial communities), but high rate 491 of microbial dispersal can also homogenize microbial communities thereby contributing little to 492 community differentiation (along cPCoA1) relative to other functional groups (Figure 4c,d). In 493 contrast, the effect of visitation by true bugs and flies on microbial community differentiation 494 may reflect microbial dispersal limitation mediated by these abundant functional groups.

495 Whether dispersal limitation is caused by shorter travel distance among flowers or fewer 
microbes being transported and delivered by true bugs and flies relative to bees needs further investigation. In addition to the distinct effects among pollinator functional groups, the same functional group can affect bacterial and fungal communities differently. For instance, bees tended to increase bacterial but decrease fungal $\alpha$-diversity. While not affecting pollinator visitation (see discussion below), resident microbes may affect whether and how much pollinators sample and consume nectar with fungi given their preference for fungi (e.g. yeasts) over bacteria (Good et al., 2014; Vannette et al., 2013). This may lead to the negative net outcome of pollinator visitation on fungal diversity but positive effect on bacterial diversity. Taken together, it suggests that diverse insect pollinators can influence the key properties of bacterial and fungal communities.

Pollinator-mediated dispersal did not act alone but interacted with other processes in driving floral microbiome. Contrary to the expectation of off-target effects on pollinators of agrochemical use (Park et al., 2015; Stejskalová et al., 2018), visitation of most functional groups did not respond to bactericide and/or fungicide. Rather pollinator visitation was strongly

511 influenced by flower abundance that signals resource availability, in agreement with the

512 observations across a broad range of plant lineages in natural ecosystems (Benadi \& Pauw, 2018;

513 Wei et al., 2020). Here, flower abundance influenced not only pollinator-mediated microbial

514 dispersal but it also had direct effect on microbiome properties suggesting it influenced the

515 source pool of microbes. The direct and indirect effect of flower abundance underscored the

516 importance of considering this driver in the principles of microbiome assembly in flowers.

Although floral microbes have been implicated in mediating plant-pollinator interactions (reviewed in Vannette, 2020), our results showed little evidence. This is because while microbes were significantly reduced, pollinator visitation remained unaffected under fungicide

522 seem less or more attractive to pollinators. The discrepancy with previous studies likely has three 523 reasons. First, studies to date (reviewed in Vannette, 2020) often used artificial flowers and/or 524 nectar to ensure that changes in cues for pollinators were induced by microbes. But in natural 525 environments many other factors including flower abundance (Armbruster, 2017; Kantsa et al., 2018; Wei et al., 2020) can also cue the presence or quality of floral resources and thereby 
527 influence pollinator visitation, which are perhaps more salient than microbes in mediating plant-

528 pollinator interactions in nature. Second, effects on only a few pollinator taxa have been tested to 529 date (Good et al., 2014; Schaeffer et al., 2019; Vannette et al., 2013), so it remains an open

530 question whether and how taxonomically diverse pollinators respond to floral microbes. Lastly,

531 previous studies that focused on one or a few microbes (reviewed in Vannette, 2020) neglected

532 complex microbe-microbe interactions in natural microbiomes as seen here. Thus it merits

533 further investigation whether findings based on a few microbes are generalizable to natural

534 microbiomes consisting of thousands or more taxa of bacteria and fungi in many flowering 535 plants.

537 Agrochemical disturbance from fungicide directly affected floral microbiome, consistent with 538 the observations in other systems and/or plant organs (Bartlewicz et al., 2016; Debode, Van 539 Hemelrijck, Creemers, \& Maes, 2013; Gu et al., 2010; Schaeffer et al., 2017). Although 540 oxytetracycline is among the most widely used bactericides (McManus et al., 2002; Vidaver, 541 2002), it caused non-significant reduction in bacterial $\alpha$-diversity and mild changes in 542 composition such as Hymenobacter, the common bacteria in strawberry flowers (Wei \& 543 Ashman, 2018). Yet, we could not rule out that it may reduce the absolute abundance of bacteria, 544 given that sequencing-based characterization of microbiomes considered only relative 545 abundances. In contrast to the bactericide, azoxystrobin, one of the most popular fungicides that 546 target eukaryotes (Battaglin, Sandstrom, Kuivila, Kolpin, \& Meyer, 2010), affected both fungal 547 and bacterial communities. It is perhaps not surprising that fungicide affected fungal 548 communities (Bartlewicz et al., 2016; Debode et al., 2013; Gu et al., 2010; Schaeffer et al., 549 2017). But the strong effect of fungicide on bacterial communities underscored important inter550 kingdom interactions. Fungi-bacteria interactions have been found predominantly negative in 551 rhizosphere (Duran et al., 2018), but these interactions can be context dependent and shift in 552 directions from negative to more positive in resource-limited environments (Velez et al., 2018), 553 such as anthosphere here (Figure 3e). The observed positive fungi-bacteria interactions likely 554 had physical (e.g. habitat sharing via fungal hyphae and bacterial biofilm) and/or metabolic 555 reasons (e.g. by-product cross-feeding) (Deveau et al., 2018; Frey-Klett et al., 2011). More 556 importantly, microbe-microbe interactions were beyond pairwise but formed a complex network, 557 leading to cascading effects of agrochemical disturbance via network links. Therefore, reducing 
558 fungi affected not only positive fungi-bacteria but also positive bacteria-bacteria interactions

559 (Figure 3e). Overall, our results indicated complex species interactions underlying how floral

560 microbiome responded to agrochemical disturbance.

562 In conclusion, the functional links between pollinator-mediated dispersal, flower abundance and

563 agrochemical disturbance improve the mechanistic understanding of microbiome assembly in

564 flowers. These findings may be generalizable to many other plants in natural and managed

565 ecosystems, as we considered not only a plant species visited by diverse insect pollinators but

566 also the common agrochemical disturbance (Battaglin et al., 2010; Vidaver, 2002) that plants

567 may encounter. In light of agricultural intensification (Matson et al., 1997; McManus et al.,

568 2002) and pollinator loss worldwide (Potts et al., 2010), it becomes more urgent than ever to

569 understand how these anthropogenic changes in plant-pollinator interactions and disturbance

570 alter microbiomes of on- and off-target plant species. 
(a)

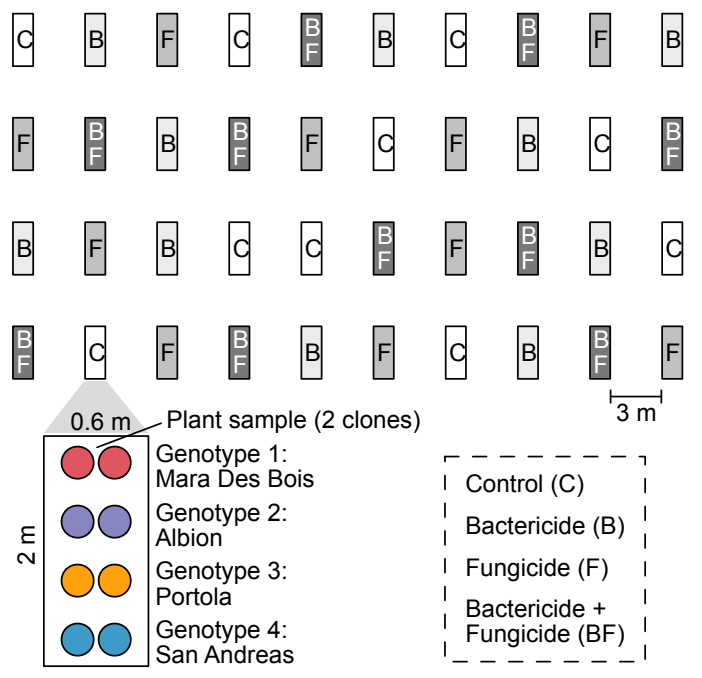

(b)

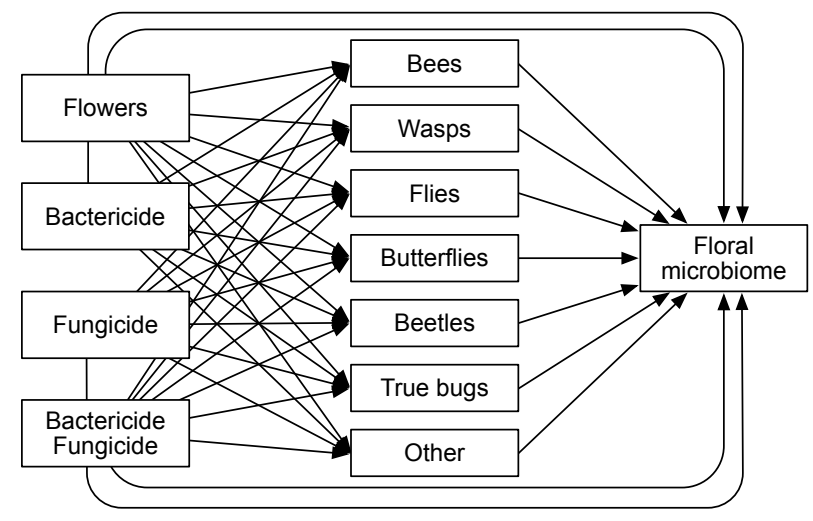

574 Figure 1 Summary of experimental design and hypotheses in structural equation modeling. (a)

575 Completely randomized, replicated factorial design was used to examine how pollinators influence floral microbiome under agrochemical disturbance. Each treatment had 10 replicates

577 ('blocks'). Each block consisted of four strawberry genotypes, with two clonal plants per

578 genotype (referred to as one plant sample) to increase floral display for pollinators. Blocks were spaced to minimize potential treatment spillover. (b) Hypothesized effects of flower abundance and agrochemical disturbance on pollinator visitation and floral microbiome. Although floral

581 microbiome may also influence pollinator visitation, this effect was unlikely in this study (see 582 main text) and thus one-way arrows were used to represent the effect of pollinator visitation on

583 floral microbiome. Floral microbiome was characterized using bacterial and fungal $\alpha-$ and $\beta$ -

584 diversity and microbial network centrality here. 
(a)

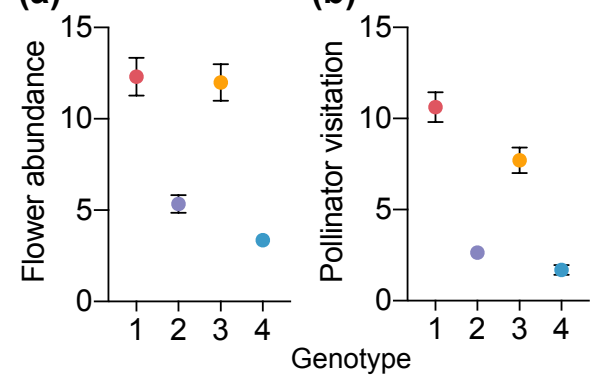

(c)

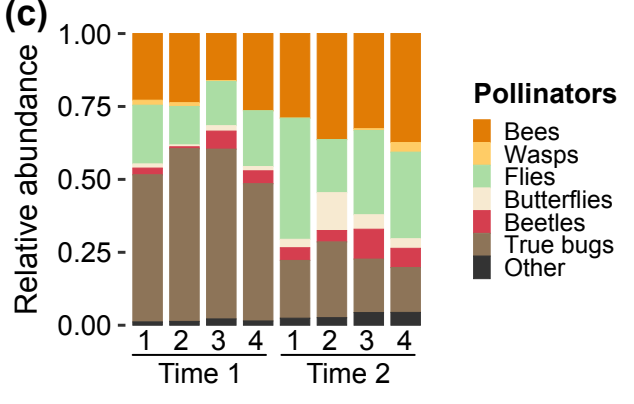

588 Figure 2 Plant genotypes differ in flower abundance and visting pollinators. Genotypic variation

589 in (a) flower abundance matched (b) pollinator visitation (i.e. number of visiting pollinators per

$590 \sim 36 \mathrm{~min}$ ) per plant sample during a 2-wk time period. (a and b) The least-squares means are

591 plotted with error bars (1 SE) after controlling for agrochemical treatment and time period (the

592 first vs. last 2 wk, Time 1 vs. Time 2) in generalized linear mixed models (Tables S1 and S2). (c)

593 Pollinator community composition changed significantly over the two time periods and among

594 genotypes (Table S2). The four strawberry genotypes were: Mara Des Bois (1), Albion (2),

595 Portola (3), and San Andreas (4). 

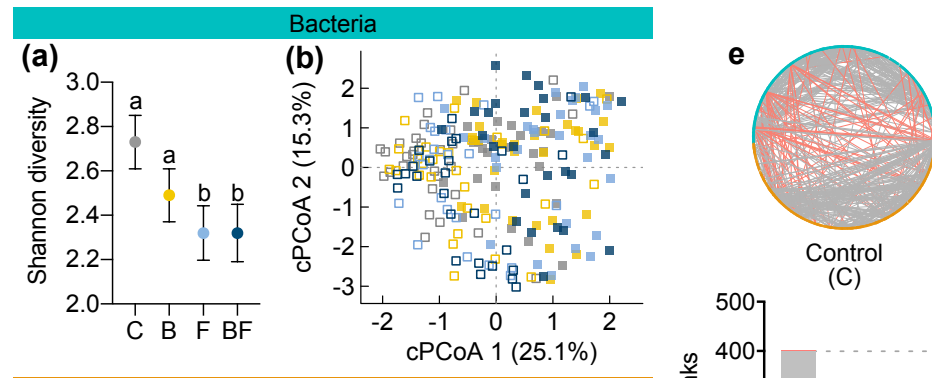

Contro
(C)
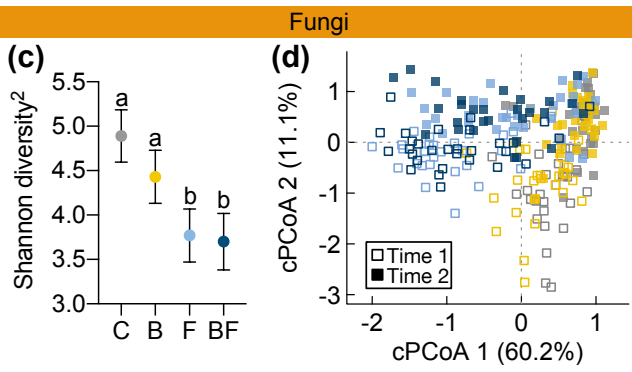

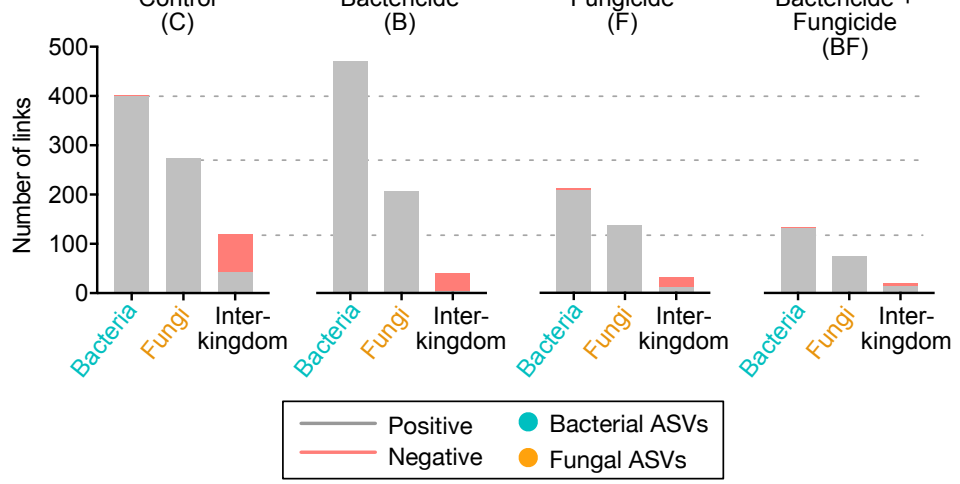

Figure 3 Microbial $\alpha$ - and $\beta$-diversity and network respond to agrochemical disturbance. The least-squares means of Shannon diversity for bacteria (a) and fungi (b; power-transformed, power parameter $=2)$ are plotted with error bars $(1 \mathrm{SE})$ for each treatment $(\mathrm{C}$, control; $\mathrm{B}$, bactericide; $\mathrm{F}$, fungicide; $\mathrm{BF}$, bactericide and fungicide), after controlling for the effects of genotype and time period in general linear mixed models (Table S4). (b and d) Constrained principal coordinates analyses (cPCoAs) indicated microbial community separation by agrochemical treatment (color) and time period (the first vs. last $2 \mathrm{wk}$, Time $1 \mathrm{vs.} \mathrm{Time} \mathrm{2)} \mathrm{for}$ bacteria (b, $N=223$ samples) and fungi (d, $N=240$ ). (e) Microbial correlation networks were based on SparCC (correlation estimates $\geq 0.4$ or $\leq-0.4)$. The same set of bacterial $(N=359)$ and fungal $(N=343)$ amplicon sequence variants (ASVs) are represented as nodes in the networks

610 across treatments (e, top panel). Positive (grey) and negative (red) intra-kingdom (bacteria-

611 bacteria and fungi-fungi) and inter-kingdom (fungi-bacteria) correlations are represented as

612 links within the networks (e, top panel). The number of these microbial correlations (links) are 613 summarized (e, bottom panel). 
(a)

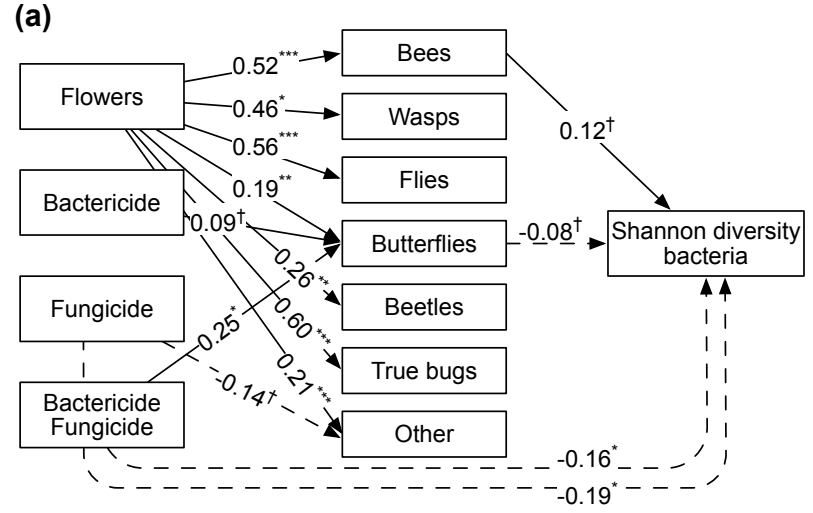

(c)

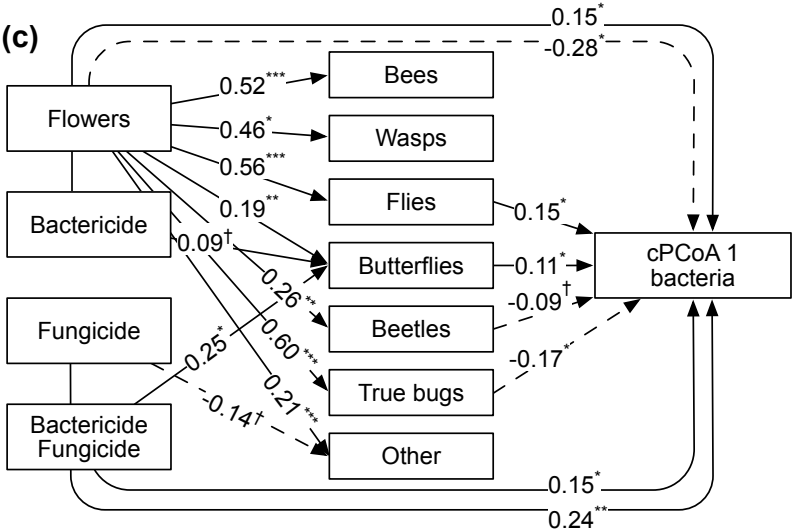

(e)

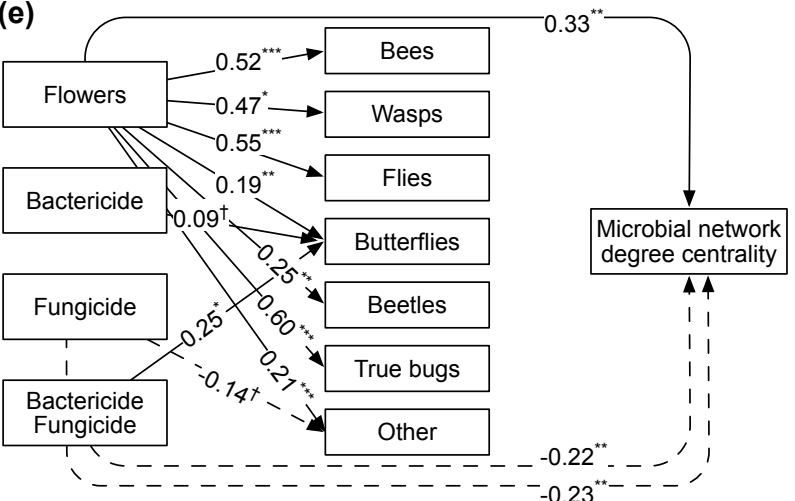

(b)

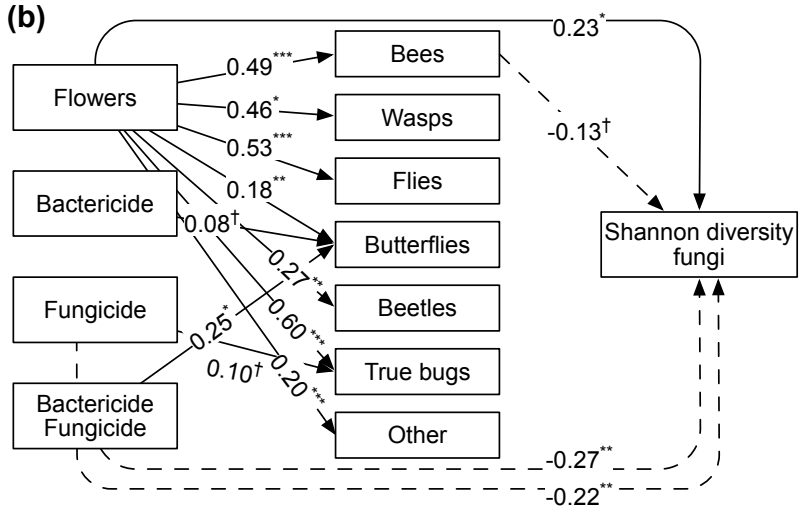

(d)

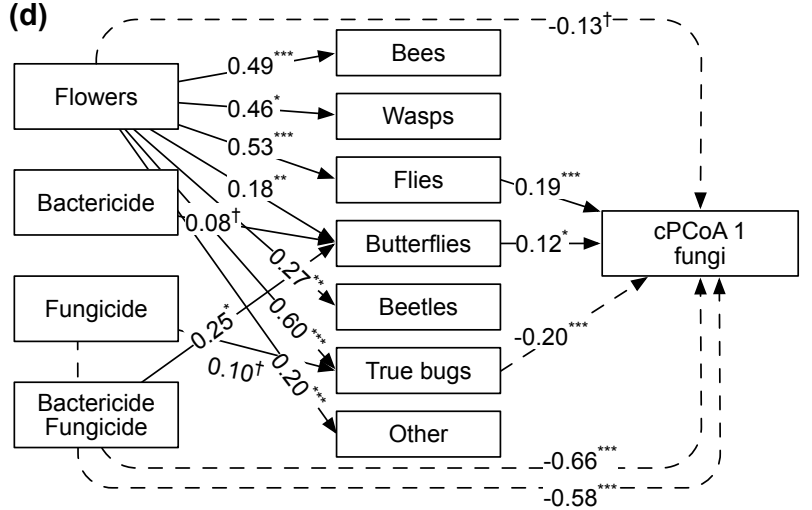

(f)

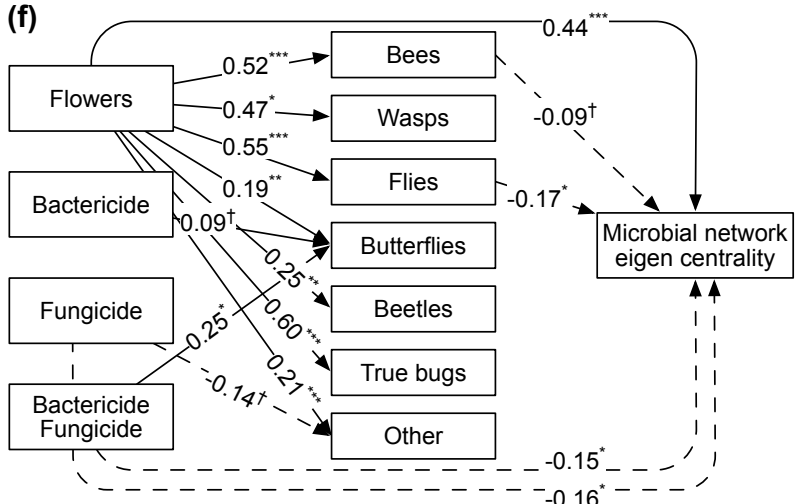

618 Figure 4 Structural equation models of flower abundance and agrochemical disturbance explaining pollinator visitation and floral microbiome. Arrows indicate notable positive (solid)

620 and negative (dashed) relationships: $\uparrow P<0.10 ; * P<0.05 ; * * P<0.01 ; * * * P<0.001$. Numbers

621 adjacent to arrows indicate standardized path coefficients. Agrochemical treatments were coded 622 using the control treatment as the reference level in the structural equation models (SEMs). The $623 \alpha$ - and $\beta$-diversity of bacterial and fungal communities used Shannon diversity ( $a$ and $b$ ) and the

624 first axis of constrained principal coordinates analysis (cPCoA1, c and d; see also Figure 3), 
625 respectively. Microbial network was built on a reduced set of amplicon sequence variants

626 (ASVs; $N=238,146$ bacteria and 92 fungi) using maximal information coefficient (MIC $\geq 0.2$;

627 see Methods for details). (e) The degree centrality and (f) eigenvector centrality measure network

628 importance of individual ASVs based on the number of direct interactions and the weighted sum

629 of both direct and indirect interactions in a network, respectively. Details in SEM model fit and

630 parameter estimation are described in Table S6.

631 


\section{References}

Albanese, D., Filosi, M., Visintainer, R., Riccadonna, S., Jurman, G., \& Furlanello, C. (2013). Minerva and minepy: a $\mathrm{C}$ engine for the MINE suite and its R, Python and MATLAB wrappers. Bioinformatics, 29, 407-408.

Aleklett, K., Hart, M., \& Shade, A. (2014). The microbial ecology of flowers: an emerging frontier in phyllosphere research. Botany, 92, 253-266.

Armbruster, W. S. (2017). The specialization continuum in pollination systems: diversity of concepts and implications for ecology, evolution and conservation. Functional Ecology, 31, 88-100.

Ashman, T.-L. (2000). Pollinator selectivity and its implications for the evolution of dioecy and sexual dimorphism. Ecology, 81, 2577-2591.

Bartlewicz, J., Pozo, M. I., Honnay, O., Lievens, B., \& Jacquemyn, H. (2016). Effects of agricultural fungicides on microorganisms associated with floral nectar: susceptibility

Bates, D., Machler, M., Bolker, B. M., \& Walker, S. C. (2015). Fitting linear mixed-effects models using lme4. Journal of Statistical Software, 67, 1-48.

Battaglin, W. A., Sandstrom, M. W., Kuivila, K. M., Kolpin, D. W., \& Meyer, M. T. (2010). Occurrence of azoxystrobin, propiconazole, and selected other fungicides in US streams, 2005-2006. Water, Air, \& Soil Pollution, 218, 307-322.

Benadi, G., \& Pauw, A. (2018). Frequency dependence of pollinator visitation rates suggests that pollination niches can allow plant species coexistence. Journal of Ecology, 106, 18921901.

Berendsen, R. L., Pieterse, C. M. J., \& Bakker, P. A. H. M. (2012). The rhizosphere microbiome and plant health. Trends in Plant Science, 17, 478-486.

Bolyen, E., Rideout, J. R., Dillon, M. R., Bokulich, N. A., Abnet, C. C., Al-Ghalith, G. A., ... Caporaso, J. G. (2019). Reproducible, interactive, scalable and extensible microbiome data science using QIIME 2. Nature Biotechnology, 37, 852-857. 564. 
Brooks, M. E., Kristensen, K., van Benthem, K. J., Magnusson, A., Berg, C. W., Nielsen, A., ... Bolker, B. M. (2017). glmmTMB balances speed and flexibility among packages for zero-inflated generalized linear mixed modeling. The R Journal, 9, 378-400.

Bulgarelli, D., Schlaeppi, K., Spaepen, S., Ver Loren van Themaat, E., \& Schulze-Lefert, P. (2013). Structure and functions of the bacterial microbiota of plants. Annual Review of Plant Biology, 64, 807-838.

Callahan, B. J., McMurdie, P. J., Rosen, M. J., Han, A. W., Johnson, A. J., \& Holmes, S. P. (2016). DADA2: High-resolution sample inference from Illumina amplicon data. Nature Methods, 13, 581-583.

Csardi, G., \& Nepusz, T. (2006). The igraph software package for complex network research. InterJournal Complex Systems, 1695, 1-9.

Debode, J., Van Hemelrijck, W., Creemers, P., \& Maes, M. (2013). Effect of fungicides on epiphytic yeasts associated with strawberry. MicrobiologyOpen, 2, 482-491.

Deveau, A., Bonito, G., Uehling, J., Paoletti, M., Becker, M., Bindschedler, S., ... Wick, L. Y. (2018). Bacterial-fungal interactions: ecology, mechanisms and challenges. FEMS Microbiology Reviews, 42, 335-352.

Diaz-Uriarte, R. (2007). GeneSrF and varSelRF: a web-based tool and R package for gene selection and classification using random forest. BMC Bioinformatics, 8, 328.

Engel, P., Kwong, W. K., McFrederick, Q., Anderson, K. E., Barribeau, S. M., Chandler, J.

Environmental Working Group. (2020). EWG's 2020 Shopper's Guide to Pesticides in Produce. https://www.ewg.org/foodnews/dirty-dozen.php.

FAOSTAT. (2020). Food and Agricultural Organaziation of the United Nations. http://faostat.fao.org/.

Fisher, A., 2nd, Coleman, C., Hoffmann, C., Fritz, B., \& Rangel, J. (2017). The synergistic effects of almond protection fungicides on honey bee (Hymenoptera: Apidae) forager survival. Journal of Economic Entomology, 110, 802-808. 
Fox, J., \& Weisberg, S. (2011). An R companion to applied regression. Thousand Oaks CA: SAGE.

Frey-Klett, P., Burlinson, P., Deveau, A., Barret, M., Tarkka, M., \& Sarniguet, A. (2011).

Friedman, J., \& Alm, E. J. (2012). Inferring correlation networks from genomic survey data. PLOS Computational Biology, 8, e1002687.

Goldford, J. E., Lu, N., Bajic, D., Estrela, S., Tikhonov, M., Sanchez-Gorostiaga, A., ... Sanchez, A. (2018). Emergent simplicity in microbial community assembly. Science, 361, 469474.

Good, A. P., Gauthier, M. P., Vannette, R. L., \& Fukami, T. (2014). Honey bees avoid nectar colonized by three bacterial species, but not by a yeast species, isolated from the bee gut. PLOS One, 9, e86494.

Gu, L., Bai, Z., Jin, B., Hu, Q., Wang, H., Zhuang, G., \& Zhang, H. (2010). Assessing the impact of fungicide enostroburin application on bacterial community in wheat phyllosphere. Journal of Environmental Sciences, 22, 134-141.

Hartig, F. (2019). DHARMa: Residual diagnostics for hierarchical (multi-Level / mixed) regression models. $\mathrm{R}$ package version 0.2 .6 . https://CRAN.Rproject.org/package=DHARMa.

Hawkes, C. V., \& Connor, E. W. (2017). Translating phytobiomes from theory to practice: Ecological and evolutionary considerations. Phytobiomes Journal, 1, 57-69.

Herrera, C. M., Pozo, M. I., \& Medrano, M. (2013). Yeasts in nectar of an early-blooming herb: sought by bumble bees, detrimental to plant fecundity. Ecology, 94, 273-279.

Kantsa, A., Raguso, R. A., Dyer, A. G., Olesen, J. M., Tscheulin, T., \& Petanidou, T. (2018). Disentangling the role of floral sensory stimuli in pollination networks. Nature Communications, 9, 1041.

Klatt, B. K., Holzschuh, A., Westphal, C., Clough, Y., Smit, I., Pawelzik, E., \& Tscharntke, T. (2014). Bee pollination improves crop quality, shelf life and commercial value. Proceedings of the Royal Society B: Biological Sciences, 281, 20132440.

Kline, R. B. (2015). Principles and Practice of Structural Equation Modeling (4th ed.). New York: Guilford Publications. 
Kuhn, M. (2019). caret: Classification and Regression Training. Contributions from Jed Wing, Steve Weston, Andre Williams, Chris Keefer, Allan Engelhardt, Tony Cooper, Zachary Mayer, Brenton Kenkel, the R Core Team, Michael Benesty, Reynald Lescarbeau, Andrew Ziem, Luca Scrucca, Yuan Tang, Can Candan and Tyler Hunt. R package version 6.0-84. https://CRAN.R-project.org/package=caret.

Kurtz, Z. D., Muller, C. L., Miraldi, E. R., Littman, D. R., Blaser, M. J., \& Bonneau, R. A. (2015). Sparse and compositionally robust inference of microbial ecological networks. PLOS Computational Biology, 11, e1004226.

Kuznetsova, A., Brockhoff, P. B., \& Christensen, R. H. B. (2017). lmerTest package: Tests in linear mixed effects models. Journal of Statistical Software, 82, 1-26.

Kwong, W. K., Medina, L. A., Koch, H., Sing, K. W., Soh, E. J. Y., Ascher, J. S., ... Moran, N. A. (2017). Dynamic microbiome evolution in social bees. Sci $A d v, 3$, e1600513.

Lenth, R. (2019). emmeans: Estimated Marginal Means, aka Least-Squares Means. R package version 1.3.4. https://CRAN.R-project.org/package=emmeans.

Liaw, A., \& Wiener, M. (2002). Classification and Regression by randomForest. $R$ News 2, 1822.

Matson, P. A., Parton, W. J., Power, A. G., \& Swift, M. J. (1997). Agricultural intensification and ecosystem properties. Science, 277, 504-509.

McManus, P. S., Stockwell, V. O., Sundin, G. W., \& Jones, A. L. (2002). Antibiotic use in plant agriculture. Annual Review of Phytopathology, 40, 443-465.

McMurdie, P. J., \& Holmes, S. (2013). phyloseq: an R package for reproducible interactive analysis and graphics of microbiome census data. PLOS One, 8, e61217.

Mouillot, D., Graham, N. A., Villeger, S., Mason, N. W., \& Bellwood, D. R. (2013). A functional approach reveals community responses to disturbances. Trends in Ecology \& Evolution, 28, 167-177.

Oksanen, J., Blanchet, F. G., Friendly, M., Kindt, R., Legendre, P., McGlinn, D., ... Helene, W. (2019). vegan: Community Ecology Package. R package version 2.5-5. https://CRAN.Rproject.org/package= $=$ vegan.

Park, M. G., Blitzer, E. J., Gibbs, J., Losey, J. E., \& Danforth, B. N. (2015). Negative effects of pesticides on wild bee communities can be buffered by landscape context. Proceedings of the Royal Society B: Biological Sciences, 282, 20150299. 
Potts, S. G., Biesmeijer, J. C., Kremen, C., Neumann, P., Schweiger, O., \& Kunin, W. E. (2010). Global pollinator declines: trends, impacts and drivers. Trends in Ecology \& Evolution, $25,345-353$.

R Core Team. (2019). R: A language and environment for statistical computing. Vienna, Austria: R Foundation for Statistical Computing. https://www.R-project.org/.

Rebolleda-Gómez, M., \& Ashman, T.-L. (2019). Floral organs act as environmental filters and interact with pollinators to structure the yellow monkeyflower (Mimulus guttatus) floral microbiome. Molecular Ecology, 28, 5155-5171.

Rebolleda-Gómez, M., Forrester, N. J., Russell, A. L., Wei, N., Fetters, A. M., Stephens, J. D., \& Ashman, T.-L. (2019). Gazing into the anthosphere: considering how microbes influence floral evolution. New Phytologist, 224, 1012-1020.

Redford, A. J., Bowers, R. M., Knight, R., Linhart, Y., \& Fierer, N. (2010). The ecology of the phyllosphere: geographic and phylogenetic variability in the distribution of bacteria on tree leaves. Environmental Microbiology, 12, 2885-2893.

Reshef, D. N., Reshef, Y. A., Finucane, H. K., Grossman, S. R., McVean, G., Turnbaugh, P. J., ... Sabeti, P. C. (2011). Detecting novel associations in large data sets. Science, 334, 1518-1524.

Rosseel, Y. (2012). Lavaan: An R package for structural equation modeling. Journal of Statistical Software, 48, 1-36.

Russell, A. L., Rebolleda-Gómez, M., Shaible, T. M., \& Ashman, T.-L. (2019). Movers and

Schaeffer, R. N., Rering, C. C., Maalouf, I., Beck, J. J., \& Vannette, R. L. (2019). Microbial metabolites elicit distinct olfactory and gustatory preferences in bumblebees. Biology Letters, 15, 20190132. shakers: Bumble bee foraging behavior shapes the dispersal of microbes among and within flowers. Ecosphere, 10, e02714. inference from next generation DNA sequencing. PLOS One, 9, e90234. 
Smith, N. W., Shorten, P. R., Altermann, E., Roy, N. C., \& McNabb, W. C. (2019). The classification and evolution of bacterial cross-feeding. Frontiers in Ecology and Evolution, 7.

Stejskalová, M., Konradyová, V., Suchanová, M., \& Kazda, J. (2018). Is pollinator visitation of Helianthus annuus (sunflower) influenced by cultivar or pesticide treatment? Crop Protection, 114, 83-89.

Vannette, R. L. (2020). The floral microbiome: Plant, pollinator, and microbial perspectives. Annual Review of Ecology, Evolution, and Systematics, 51.

Vannette, R. L., \& Fukami, T. (2017). Dispersal enhances beta diversity in nectar microbes. Ecology Letters, 20, 901-910.

Vannette, R. L., Gauthier, M. P., \& Fukami, T. (2013). Nectar bacteria, but not yeast, weaken a plant-pollinator mutualism. Proceedings of the Royal Society B: Biological Sciences, 280, 20122601.

Vannette, R. L., Hall, G., \& Munkres, I. (2020). Fungi are more dispersal-limited than bacteria among flowers. bioRxiv, https://doi.org/10.1101/2020.1105.1119.104968.

Velez, P., Espinosa-Asuar, L., Figueroa, M., Gasca-Pineda, J., Aguirre-von-Wobeser, E., Eguiarte, L. E., ... Souza, V. (2018). Nutrient dependent cross-kingdom interactions: Fungi and bacteria from an oligotrophic desert oasis. Frontiers in Microbiology, 9, 1755.

Vorholt, J. A. (2012). Microbial life in the phyllosphere. Nature Reviews Microbiology, 10, 828840.

Wei, N., \& Ashman, T.-L. (2018). The effects of host species and sexual dimorphism differ among root, leaf and flower microbiomes of wild strawberries in situ. Scientific Reports,

Wei, N., Kaczorowski, R. L., Arceo-Gómez, G., O’Neill, E. M., Hayes, R. A., \& Ashman, T.-L. (2020). Pollinator niche partitioning and asymmetric facilitation contribute to the maintenance of diversity. bioRxiv, https://doi.org/10.1101/2020.1103.1102.974022. 